\title{
Neuromodulación eléctrica y dolor raquídeo
}

\author{
G. García-March ${ }^{1}$ y G. García-Catalán ${ }^{2}$

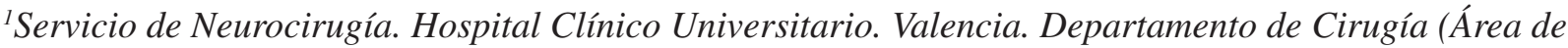 \\ Neurocirugía). Facultad de Medicina y Odontología. Universidad de Valencia. ${ }^{2}$ Facultad de Medicina. \\ Universidad Católica de Valencia. Valencia
}

García-March G, García-Catalán G. Neuromodulación eléctrica y dolor raquídeo. Rev Soc Esp Dolor 2015; 22(3): 116-125.

\begin{abstract}
The pain of spinal origin is a pathological condition with a significant incidence and prevalence and enormous social and health impact. The different electrical neuromodulation techniques can be considered an effective and safe alternative for the treatment of these patients. In the present review, we deep from the etiological and pathophysiological in spinal pain, especially in the syndrome of failed spinal surgery. We describe the surgical implantation technique, its indications and results achieved until now. We will also summarize the possible mechanisms of action of different types of electrical neurostimulation. Finally, we will comment on the dificulties and inconvinients that did not allow this pain surgical alternative to become more widely used.
\end{abstract}

Key words: Spinal pain. Physiopathology. Electric neuroestimulation. Techniques and results. Mechanisms of action.

\section{RESUMEN}

El dolor de origen raquídeo es un cuadro patológico con una importante incidencia y prevalencia y un enorme impacto socio-sanitario. La incorporación de las diferentes técnicas de neuromodulación eléctrica en el tratamiento de estos pacientes se ha mostrado como una alternativa terapéutica novedosa, segura y eficaz. El presente trabajo de revisión pretende profundizar desde el punto de vista etiológico y fisiopatológico en el dolor raquídeo, y sobre todo en el síndrome de cirugía fallida espinal. Se intentará acercar la técnica quirúrgica de implantación, comentar las indicaciones y resultados obtenidos hasta la actualidad, y realizar una aproximación crítica a los posibles mecanismos de acción de las diferentes modalidades de neuroestimulación eléctrica. Finalmente, se comentarán cuáles han sido los problemas e inconvenientes que han impedido la utilización generalizada de esta alternativa quirúrgica en estos procesos.

Palabras clave: Dolor raquídeo. Fisiopatología. Neuroestimulación eléctrica. Técnicas y resultados. Mecanismos de acción.

\section{INTRODUCCIÓN}

El dolor de origen raquídeo es un cuadro patológico con una importante incidencia y prevalencia. Se estima que un $80 \%$ de la población lo sufre en algún momento de su vida, concretándose en diferentes estudios que la prevalencia de lumbalgia crónica entre los adultos españoles es del 7,7\%, y que la incidencia de la población que acude a una Unidad de Dolor en España por problemas de dolor raquídeo no oncológico es del 55,3\% $(1,2)$. Es además un trastorno con un enorme impacto socio-sanitario, con importantes repercusiones psicológicas y económicas tanto en el paciente que lo sufre como en la sociedad que tiene que sufragar los gastos que el mismo supone.

En la actualidad existe un enorme abanico de posibles soluciones quirúrgicas a los diferentes problemas raquídeos que, en general, deben ser abordados de forma multidisciplinar. Sin embargo queda un grupo de pacientes con dolor en los que la cirugía convencional no ofrece 
garantías suficientes de eficiencia y seguridad (o bien esta ha fracasado) y que demanda solución a sus problemas. Es precisamente en estos grupos donde se están buscando nuevas alternativas terapéuticas y, entre ellas, la estimulación eléctrica de forma crónica de diferentes estructuras nerviosas ha resultado ser de las más novedosas, seguras y eficaces.

\section{ETIOPATOGENIA Y FISIOPATOLOGÍA}

El mecanismo de producción del dolor de origen espinal es complejo, ya que sobre causas primarias y secundarias se suman factores psicosociales que pueden jugar su papel. Las causas primarias de dolor espinal pueden ser mecánicas cuando el foco doloroso se localiza en alguno de los componentes del complejo tri-articular raquídeo, o radiculares, incluyendo la hernia discal, la estenosis de canal, la estenosis del receso lateral, la fibrosis epidural y la aracnoiditis. Una vez iniciado el dolor por una de estas causas se produce una serie de mecanismos secundarios que cronifican el estímulo nociceptivo a través de un círculo vicioso de espasmos musculares e hiperactividad del sistema simpático. Sobre este complejo entramado los factores psicosociales pueden actuar produciendo una disfunción en las vías de inhibición descendentes del sistema nervioso, cronificándose el dolor y desapareciendo el concepto clásico de causa-efecto en la producción del mismo. Se hace así difícil la explicación de la etiopatogenia y fisiopatalogía al no existir una correlación entre las alteraciones estructurales raquídeas y el dolor espinal (3).

Comentario aparte merece el síndrome de cirugía fallida de espalda, término no específico que describe el dolor crónico persistente que aparece entre el $10-40 \%$ de pacientes sometidos previamente a cirugía lumbar $(4,5)$. En general se acepta que se trata de un dolor lumbar con o sin compromiso radicular, con o sin irradiación a miembros inferiores, persistente o recurrente, que se presenta tras una cirugía correctamente realizada de columna lumbar y donde no se evidencia ninguna patología macroscópica que justifique una reintervención $(6,7)$. Aunque este síndrome generalmente se ha asociado a la columna lumbar, cada vez se ven más pacientes con problemas en raquis cervical y extremidades superiores tras la cirugía a esos niveles y que también podrían ser englobados dentro de esta entidad. Habitualmente se ha relacionado con una mala selección de los pacientes con una indicación poco acertada o incorecta debido a una difícil valoración de la clínica, severidad y localización del dolor, a una cirugía inadecuada (nivel equivocado o mala técnica) o a complicaciones de la cirugía o de los procedimientos diagnósticos (fibrosis peridural o aracnoiditis postquirúrgica). En la etiopatogenia del síndrome se suelen reconocer estenosis, degeneración de los segmentos adyacentes, hernia de disco recurrente, fragmentos de disco retenidos, espondilolistesis, fibrosis, enfermedad discal degenerativa, radiculopatía, dolor discal, dolor facetario, dolor sacroiliaco, discitis, aracnoiditis, pseudoartrosis e inestabilidad segmentaria. La fibrosis epidural está presente en un 20-36\% de los pacientes (7). En todos estos casos, los mecanismos fisiopatológicos que se desencadenan producen una serie de modificaciones que conducen a una sensibilización del sistema nervioso central y periférico, que es a su vez potenciada por el daño inflamatorio y la injuria nerviosa. La persistencia del estímulo nociceptivo provoca una liberación masiva de glutamato y sustancia $\mathrm{P}$ en la médula espinal, lo que conlleva a unas modificaciones en el asta dorsal medular con liberación de quimoquinas e interleucinas que van a activar la microglía y a los astrocitos. Esta activación glial se induce también por la presencia de sustancias endógenas denominadas alarmitas, la fibronectina y proteínas de choque térmico o la presencia de ATP. La respuesta glial en estos casos será la liberación masiva de sustancias proinflamatorias que cronificarán aún más el proceso (8).

Se trata de un dolor complejo en donde coexisten el dolor neuropático, en la medida en la que se hayan lesionado en mayor o menor intensidad estructuras nerviosas a esos niveles, y el dolor nociceptivo en cuanto a que se están irritando y estimulando constantemente nociceptores subcutáneos y profundos de alrededor de la herida quirúrgica, lo que explicaría el dolor axial de la mayoría de estos síndromes. Frecuentemente se ve implicado en estos casos el sistema nervioso simpático.

Los pacientes con síndrome de cirugía fallida de espalda pueden sufrir un dolor exclusivamente nociceptivo, normalmente en la región lumbar o cervical, o un dolor neuropático, generalmente referido a varios dermatomos de una o varias extremidades. Sin embargo lo más común es una combinación de los dos, de tal forma que la situación más frecuente es la de un paciente que asocia un dolor nociceptivo en raquis lumbar y un dolor neuropático irradiado a lo largo de una o las dos extremidades inferiores.

De todo lo expuesto hasta aquí se puede concluir que el dolor raquídeo residual es una patología habitual, compleja, cuyo abordaje suele ser difícil y su tratamiento con frecuencia frustrante, que suele asociar en los pacientes problemas laborales, psicológicos, sociales y económicos. Se impone por tanto un enfoque multidisciplinar con tratamientos que se realizan en escalonamiento progresivo y donde las técnicas de neuromodulación serían el último escalón terapéutico.

\section{TÉCNICAS DE NEUROESTIMULACIÓN ELÉCTRICA}

Para que el sistema nervioso pueda desempeñar su control sobre el funcionalismo orgánico se requiere un perfec- 
to equilibrio entre los mecanismos facilitadores e inhibidores. Cuando este balance se descompensa, por exceso o por defecto, surge la patología funcional de los sistemas. Así, desde una perspectiva teórica, sería posible mediante una medida terapéutica adecuada tendente a incrementar o reducir la función, devolver el equilibrio perdido recobrándose de nuevo la eurritmia. Este fue y es el objetivo que pretende la neurocirugía funcional. Tradicionalmente, esto se cumplía con la aplicación de métodos lesivos en las zonas de control nervioso de las funciones alteradas. Sin embargo, la irreversibilidad de las lesiones, los efectos secundarios y la resistencia de ciertos procesos funcionales a estos procedimientos, plantearon un nuevo enfoque al tratamiento de estas disfunciones basado en la potenciación de los mecanismos fisiológicos de control, incrementando la facilitación o reduciendo la inhibición en los procesos hipofuncionales o el mecanismo contrario en los hiperfuncionales. Surge así, con la aplicación de estímulos eléctricos en diferentes estructuras nerviosas, el moderno capítulo terapéutico de la neuroestimulación.

Aunque en la actualidad la neuroestimulación eléctrica se ha usado en diversas vertientes del diagnóstico y terapéutica neuroquirúrgicas (epilepsia, espasticidad y movimientos anormales, enfermedad de Parkinson, trastornos psiquiátricos, electromicción medular en la paraplejia postraumática y otras disfunciones vesicales neurogénicas, entre otras) su papel terapéutico decisivo lo demostró en el campo del dolor crónico rebelde.

El uso de la electricidad en los procesos dolorosos, aunque hoy suena como novedoso, era ya conocido y utilizado en la antigüedad clásica. Así, en el año 47 d. C., Scribonius Largus recoge un caso de artritis que mostró un alivio parcial de su cuadro álgido tras el contacto accidental con un pez torpedo. Desde entonces y en estos primeros tiempos, las descargas eléctricas de esta especie animal se emplearon para tratar los dolores artrálgicos y las cefaleas (9). En la Edad Media, Avicena y Averroes continuaron recomendando su aplicación en los mismos casos (10). Fue en el siglo XVIII cuando ya se introduce la electricidad artificial producida por generadores electrostáticos en la terapéutica médica, aunque todavía con fundamentos empíricos. Con la aparición de las botellas de Leiden se multiplican las indicaciones, pero no progresan los resultados. El efecto analgésico de este tipo de estimulación recibió un amplio reconocimiento en el siglo XIX. En su principio, Duchenne de Bologne y Charles Bew tratan con descargas farádicas el dolor facial atípico y la neuralgia trigeminal; mientras en su mitad, Hermel comenzó a tratar con corriente galvánica los dolores ciáticos y las neuralgias lumbosacras (10).

El verdadero auge de la neuroestimulación aplicada al dolor surge a partir de 1965 y se desarrolla en la década de los setenta. Varios han sido los hechos que justifican esta expansión. Por un lado, el diseño de la teoría de los mo- delos inhibitorios de la transmisión del dolor de Melzack y Wall (11); por otro lado el descubrimiento de los sistemas endorfinérgicos $(12,13)$; el mejor conocimiento de la fisiopatología del dolor; y finalmente, los buenos resultados obtenidos en la clínica humana. Así, Wall y Sweet (14) proponen en 1967 la estimulación eléctrica directa de los nervios periféricos para tratar la disestesia dolorosa de los miembros. Esta técnica pretendía aumentar el influjo epicrítico sobre el control medular, a través de las fibras mielínicas, para incrementar su poder inhibidor. Simultáneamente, Shealy y cols. (15) plantean la posibilidad de estimular a largo plazo los cordones posteriores de la médula en diferentes cuadros dolorosos que afectaban a la mitad inferior del cuerpo, pensando que así se produciría por un lado una facilitación antidrómica de los sistemas medulares inhibitorios, y, por otro, un aumento de los influjos aferentes epicríticos que llegan a los mecanismos inhibitorios centrales del dolor, tratando de conseguir de esta forma un incremento de su poder inhibitorio. A partir del año 1972 y con la introducción de los electrodos percutáneos, que simplificaban el procedimiento y reducían sus complicaciones, la estimulación medular se ha relanzado lo que la ha llevado a su generalización en el momento actual.

La aceptación de la teoría de los modelos inhibidores, el avance tecnológico y los buenos resultados obtenidos con las técnicas descritas posibilitaron el desarrollo de otros métodos de estimulación, sobre todo a nivel central, para el tratamiento del dolor. En esta línea, Mazars y cols. (16) introducen la estimulación eléctrica del núcleo ventral posterior talámico en el tratamiento de procesos dolorosos por deaferentización localizados en la región cervicoorofacial. Durante el mismo periodo Richardson y Akil (17) introducen la estimulación directa del mecanismo inhibidor central mesencefálico, a base de implantar electrodos multipolares en la sustancia gris periacueductal y en la sustancia reticular periventricular, indicando que los mejores resultados se producían en procesos producidos por exceso de nocicepción, fundamentalmente en aquellos relacionados con el cáncer y la aracnoiditis. Finalmente, otros autores han utilizado la estimulación eléctrica de otros puntos diana intracerebrales, como el núcleo caudado y la región septal, con fines antiálgicos, sobre todo en casos de miembro doloroso fantasma con componente emocional (18). El descubrimiento y descripción del sistema EMA (Endorphin Mediated Analgesia System), así como el de diferentes sustancias neuromoduladoras con capacidad analgésica han revolucionado y potenciado el campo de la neuroestimulación (19).

En definitiva, hoy se puede afirmar que en mayor o menor medida, todas las modalidades de estimulación nerviosa están basadas en la potenciación de los mecanismos fisiológicos inhibidores de la transmisión nociceptiva según los postulados de Melzack y Wall. En los siguientes 
apartados se detallan las diferentes técnicas, definiéndolas, haciendo una aproximación crítica a su posible mecanismo de acción y mostrando sus resultados en el tratamiento del dolor raquídeo.

\section{Estimulación eléctrica transcutánea}

Representa uno de los métodos de inhibición del dolor más sencillos e inocuos. Consiste básicamente en la aplicación de una corriente eléctrica a través de unos electrodos emplazados directamente sobre la superficie cutánea. El verdadero auge de esta técnica se produce en la década de los 60, debido fundamentalmente a la expansión de la acupuntura oriental fuera de su ámbito y al desarrollo de los mecanismos fisiopatológicos de inhibición de la transmisión nociceptiva a nivel segmentario y central (11). Inicialmente este tipo de estimulación se aplicó como una prueba predictiva previa a la implantación crónica de electrodos medulares, suponiendo que los casos resistentes no obtendrían ninguna mejoría con la estimulación medular. Sorprendentemente, muchos casos de dolor crónico ya sólo con esta prueba conseguían resultados analgésicos satisfactorios a largo plazo. Fue así como la estimulación transcutánea se erigió como una alternativa terapéutica frente al dolor de diversa etiología.

En la actualidad ya son numerosos los estudios sobre el efecto paliativo de la estimulación transcutánea en diferentes estados de dolor agudo o crónico, siendo escasas las patologías causantes de dolor en que no se haya aplicado. En general se recogen índices de mejoría que oscilan entre el 25 y el $75 \%$ de los casos tratados $(20,21)$.

La estimulación eléctrica transcutánea requiere un estimulador eléctrico, constituido por un generador de pulso y un amplificador, y un sistema de electrodos planos a emplazar sobre la zona dolorosa. La señal eléctrica producida por el generador es amplificada hasta un nivel suficiente para descargarse a través de los electrodos, estando ello en función de la impedancia de los mismos y del tejido que los separa del nervio. Los electrodos utilizados son de carbón-silicona, flexibles y de diferentes superficies capaces de adaptarse a la superficie corporal. Un generador apropiado debe disponer de una gama de parámetros eléctricos que permitan una estimulación con una corriente de 0 a $60 \mathrm{~mA}$ de intensidad, una frecuencia de 0 a $150 \mathrm{~Hz}$ y una duración de pulso de 0,1 a $0,5 \mathrm{mseg}$. Las ondas de pulso que han resultado más efectivas en el control del dolor mediante este tipo de estimulación son las bifásicas y las rectangulares.

El mecanismo de acción de este tipo de estimulación es complejo y aun hoy no está totalmente aclarado. Se acepta que la estimulación cutánea segmentaria, vehiculada por las fibra A mielínicas, inhibe la transmisión por las fibras $\mathrm{C}$ de los impulsos dolorosos. El modo como la fibra A genera la actividad inhibitoria es desconocido, aunque se cree que ocasionaría una despolarización de los terminales de las fibras $\mathrm{C}$, tratándose así de una inhibición presináptica de la transmisión nociceptiva. Es probable además que la estimulación de las fibras A active algunas vías descendentes procedentes de centros superiores que colaboren en los mecanismos inhibitorios endorfinérgicos mediados por la serotonina. Se piensa así que podría aumentar la síntesis y liberación de opioides endógenos a nivel segmentario medular colaborando en la analgesia por un mecanismo similar al producido por la inyección intraventricular o intratecal de mórficos. Otros mecanismos implicados podrían ser el efecto galvánico de la corriente, la relajación muscular, la mejora del tropismo e incluso una acción similar al de la acupuntura $(20,22,23)$.

En el dolor agudo raquídeo puede en muchos casos sustituir o complementar la medicación analgésica, siendo útil en el $80 \%$ de los mismos (21). En el dolor crónico raquídeo, cervical, dorsal, lumbar y en casos más severos como aracnoiditis, suele ser junto las medidas físicas y psicológicas la primera aproximación terapéutica. Los índices de mejoría a largo plazo en este tipo de dolor suelen estar entre un 50-60\% de analgesia en los pacientes tratados, mientras que los resultados caen hasta un $30-40 \%$ cuando se considera como única terapéutica $(20,21)$.

La aplicación de la estimulación nerviosa transcutánea está, en líneas generales, libre de efectos colaterales. Los únicos problemas descritos son dermatitis alérgicas al gel conductor y eritemas en la zona de aplicación de los electrodos y, en algunos casos, un incremento de la sensación dolorosa durante el tratamiento. Todas estas complicaciones suelen evolucionar favorablemente al suprimir temporalmente la estimulación.

\section{Estimulación eléctrica subcutánea}

En esta novedosa modalidad terapéutica, los electrodos de cuatro u ocho contactos se implantan a nivel subcutáneo en la zona dolorosa, generalmente zonas paralaterales raquídeas, que en caso de un efecto positivo se conectarán a un generador de impulsos eléctricos similar al de otros tipos de estimulación.

Los mecanismos de acción de esta variante de neuromodulación no están todavía bien definidos, aunque parece que serían similares a los de la estimulación transcutánea o a los de la estimulación medular. La estimulación de fibras de gran tamaño $A \beta$ sería la responsable de la modulación de las aferencias derivadas de las fibras de menor tamaño A $\delta$ y C. Se postula además la modulación central a través de fibras $A \beta$ y $A \delta$ por medio de una activación anterógrada, y un efecto local en la matriz nerviosa dérmica pudiendo tener la estimulación eléctrica un efecto antiinflamatorio local directo y un efecto despolarizante a nivel 
de las membranas, con una reducción en la sensibilidad a las catecolaminas circulantes (24).

Su indicación fundamental en los casos de dolor lumbar crónico y en los síndromes de cirugía fallida de espalda es el dolor axial que no puede ser bien controlado con la estimulación medular (Fig. 1). Sus resultados cuando se aplican conjuntamente suelen ser muy beneficiosos (25).

Los efectos secundarios más habituales son el movimiento y migración del electrodo que causa cambio o pérdida de parestesias eléctricas en el área dolorosa, estimulación intermitente o molesta, dolor en el área de los electrodos o del generador y problemas en el programador con la transmisión de datos, todos ellos leves y fácilmente subsanables.

\section{Estimulación eléctrica medular}

Es la técnica de neuroestimulación más utilizada para tratar los diferentes síndromes de dolor neuropático. Basada en las teorías de Melzack y Wall (11), Shealy y cols. (15) son los primeros que introducen la estimulación eléctrica directa de los cordones posteriores en diferentes cuadros de dolor crónico. Inicialmente el procedimiento requería la colocación quirúrgica de un electrodo plano multicontacto, tras laminectomía y apertura dural sobre estos haces, aunque luego surgieron otras variantes que lo colocaban intra o epiduralmente. Los efectos secundarios inherentes a la técnica como compresión medular, fístulas de líquido cefalorraquídeo, rechazo del sistema, infección de la herida quirúrgica, dolor en la herida cutánea y, finalmente, las disfunciones del propio sistema de estimulación hizo que inicialmente no se generalizara. Los avances tecnológicos posteriores hicieron posible que en 1972 Hosobuchi y cols. (26) incorporaran el uso de electrodos cilíndricos más flexibles que se introducían en el espacio epidural por vía percutánea, lo que significó el relanzamiento definitivo de la terapia.

Básicamante se trata de estimular los cordones posteriores medulares a través del espacio epidural mediante electrodos cilíndricos multicontacto introducidos percutáneamente, o electrodos planos de varios contactos que se disponen en filas implantados mediante una pequeña laminotomía y que se van ascendiendo bajo control fluoroscópico (Fig. 2). En ambos casos y para optimizar la posición de los electrodos, se requiere que el paciente esté despierto para localizar las parestesias en la zona dolorosa. La gran ventaja de los electrodos cilíndricos es que tan sólo precisan anestesia local y una ligera sedación al implantarse percutáneamente, sin embargo tienen como inconvenien-

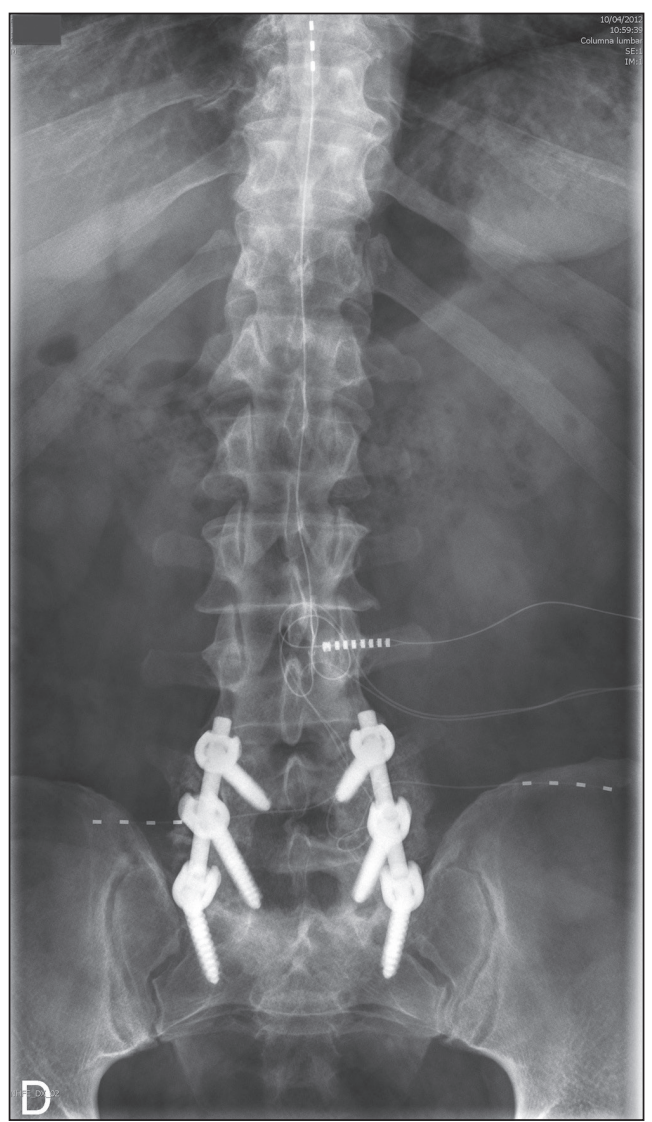

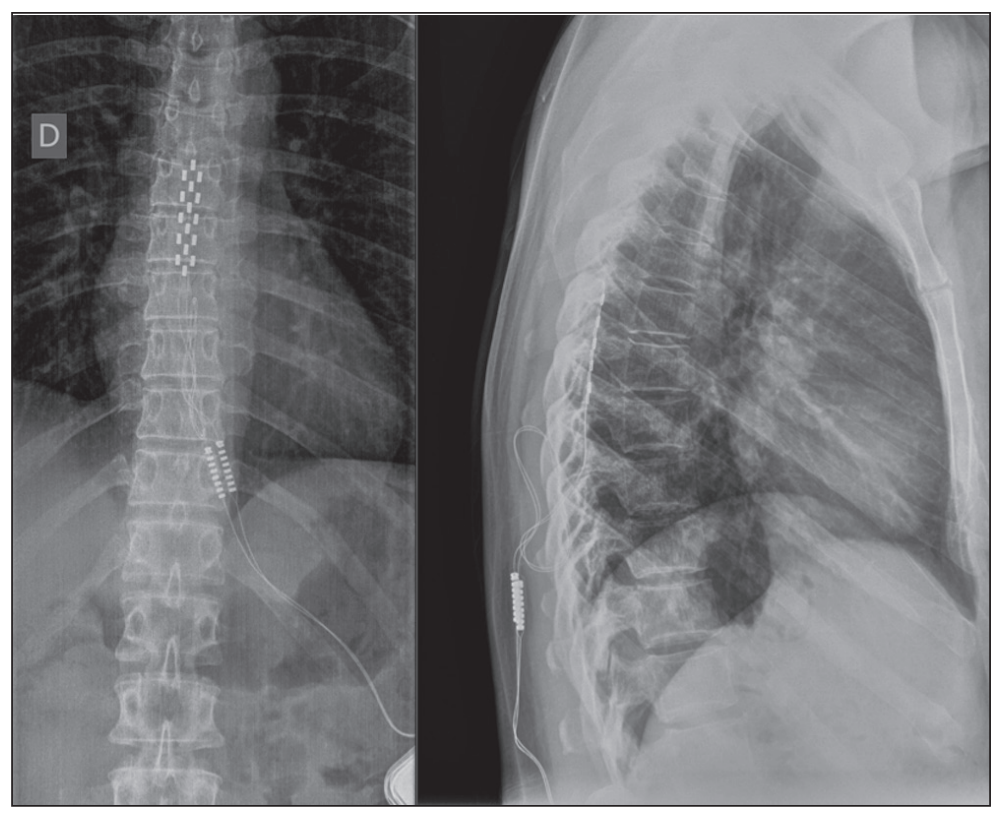

Fig. 2. Electrodo plano quirúrgico de 16 contactos para estimulación medular de localización dorsal, en proyecciones antero-posterior y lateral.

Fig. 1. Electrodo cilíndrico multicontacto para estimulación medular de localización dorsal y electrodos subcutáneos bilaterales de localización lumbar (cortesía del Prof. J.J.Pallarés). 
tes el riesgo aumentado de migración y rotura así como un requerimiento energético mayor, al tener menor contacto con la duramadre, lo que se traduce en frecuentes recambios del generador. Por el contrario, los electrodos planos quirúrgicos tienen como ventajas la facilidad y precisión en su colocación, mejor cobertura de las parestesias eléctricas sobre zonas dolorosas, menor riesgo de lesiones durales/neurales y un menor requerimiento energético. Su inconveniente más importante, la necesidad de anestesia general para realizar la laminotomía mínima para introducir el electrodo, se ha visto en la actualidad soslayado con la utilización de la raquianestesia espinal (bloqueo epidural neuroaxial) que algunos grupos, entre ellos el nuestro, están utilizando (27). La utilización de uno u otro tipo de electrodo está en función de las preferencias de cada especialista y de la localización del dolor, siendo unánime la consideración del empleo de electrodos percutáneos en los síndromes originados en zona cervical y miembros superiores. En ocasiones y en los casos de dolor raquídeo y en los de síndrome de cirugía de espalda fallida, puede que se requiera más de un electrodo percutáneo multicontacto para reproducir las parestesias eléctricas perfectamente en la zona dolorosa. En general la posición definitiva de los electrodos en el espacio epidural suele localizarse entre $\mathrm{C}_{4}$ a $\mathrm{C}_{7}$ para dolores que afecten a la zona cervical y extremidades superiores, y entre $\mathrm{Th}_{8} \mathrm{y} \mathrm{Th}_{11}$ en dolores axiales de la zona lumbo-sacra y de extremidades inferiores (28).

En caso de efecto positivo estos electrodos se conectarán a un generador que mandará impulsos eléctricos a la médula espinal. Existen en el mercado varios tipos de generadores, unos implantables no recargables (con agotamiento de la batería a los dos o tres años), otros recargables (con recarga desde el exterior por el propio paciente, con una duración media de diez años) con varios sistemas de programación de parámetros eléctricos simultáneos y otros recargables con sistemas de adaptación de los parámetros programados en función de cada posición que adopte el paciente. Los parámetros eléctricos que permiten los equipos son 0 a $10,5 \mathrm{~V}$ de intensidad de corriente, 2 a $130 \mathrm{~Hz}$ de frecuencia y de 60 a $450 \mu$ seg de ancho de pulso. Pueden funcionar en modalidad continua o cíclica. Recientemente algunos equipos han incorporado gamas más altas de frecuencias de hasta $10 \mathrm{KHz}$ con los que se está comprobando un efecto beneficioso mayor en el dolor axial y de miembros inferiores que se produce en el síndrome de cirugía fallida de espalda (29).

Los mecanismos de acción de la técnica, aunque desconocidos, pueden establecerse en torno a la teoría de la puerta de entrada, la modulación electroquímica de la transmisión neural, impulsos ortodrómicos sobre el núcleo ventral posterior talámico que activen los tractos inhibitorios descendentes, la modulación del sistema nervioso simpático o la liberación de neuromoduladores y neurotransmisores inhibidores en el asta posterior medular, incrementando los niveles de GABA, induciendo la liberación de adenosina y disminuyendo la liberación de aminoácidos excitadores como el glutamato y aspartato (30).

Los resultados en el dolor raquídeo residual y sobre todo en el dolor crónico que aparece en el síndrome de cirugía de espalda fallida suele establecerse en una analgesia de más del $50 \%$, que se mantiene a largo plazo, en el $60-80 \%$ de pacientes con radiculopatía con o sin dolor lumbar (31,32). En general en estos casos disminuye la ingesta de analgésicos opiáceos y suele asociarse a una mejoría en la calidad de vida de los pacientes, incrementando sus posibilidades de reincorporación socio-laboral (33). Algunos estudios han demostrado que la estimulación medular es más efectiva en el alivio del dolor que el rescate quirúrgico, con nueva cirugía de columna en pacientes con síndrome de cirugía fallida de espalda, con predominio de la clínica en miembros inferiores (31). Resultados similares se han recogido cuando se compara este tipo de terapia con el tratamiento conservador en estos cuadros (6). Nuestra experiencia personal recogida a lo largo de más de veinte años de tratamiento con estimulación medular en dolores por patología de origen raquídeo es similar en cuanto a resultados en todos los campos (alivio del dolor, ingesta de opiáceos, calidad de vida, reincorporación socio-laboral) al publicado por otros autores $(27,34)$. La reciente incorporación de la alta frecuencia en los sistemas de estimulación y los datos preliminares recogidos, que demuestran una disminución del dolor de la zona lumbar y de miembros inferiores, y un aumento en la calidad de vida de los pacientes, podrán dar más opciones terapéuticas en aquellos casos en los que la estimulación medular convencional no haya sido efectiva (29).

Las complicaciones más frecuentes que pueden aparecer en la estimulación medular pueden ser de orden técnico, relacionadas con la migración o fractura del electrodo, de orden biológico con la posible aparición de infecciones, fístulas de líquido cefalorraquídeo, hemorragias en el bolsillo subcutáneo del generador, y más raramente hematomas epidurales, y las relacionadas con el propio procedimiento como el dolor en el sitio de implantación y la aparición de parestesias eléctricas desagradables. En general todos estos efectos secundarios suelen ser menores y reversibles.

\section{Estimulación cerebral profunda}

La vigencia de esta técnica de neuroestimulación en el tratamiento de los cuadros de dolor crónico refractario tiene más de cincuenta años, sin embargo su auge y desarrollo tiene lugar en los años 80. Consiste en la implantación, estereotáctica o mediante técnicas de neuronavegación, de un electrodo cilíndrico multicontacto en diferentes estructuras cerebrales profundas (tálamo somatosensorial, 
cápsula interna, sustancia gris periacueductal y periventricular, hipotálamo). La elección de una u otra estructura estará en función del tipo de dolor que aparezca, así la estimulación del núcleo ventral posterior talámico y cápsula interna estará indicada principalmente en los dolores por deaferentización o neuropáticos (16), la de la sustancia gris periacueductal y periventricular en los dolores por exceso de nocicepción o mixtos (17), mientras que la del hipotálamo estaría indicada en aquellos casos de cefalea en racimo o cluster (35).

Antes de realizar cualquier modalidad de estimulación cerebral profunda se debe realizar una correcta selección del paciente, intentando identificar el tipo y localización del dolor que padece, así como los tratamientos farmacológicos o quirúrgicos antiálgicos previos. Se ha de tener en mente que este tipo de técnica junto con la que se comentará en el apartado siguiente suelen ser el último recurso a ofrecer a un paciente con dolor crónico rebelde.

La implantación del electrodo en la diana concreta se realiza mediante procedimientos estereotácticos convencionales o mediante neuronavegación con fusión de imágenes de tomografía computada y resonancia magnética cerebrales. El enfermo suele estar en todos los casos despierto. La confirmación del núcleo a estimular se hace con el registro con microelectrodos y la propia estimulación durante el tiempo quirúrgico (Fig. 3). En general se requiere que el paciente note parestesias eléctricas en la zona dolorosa sin que aparezcan efectos secundarios. Los parámetros eléctricos de estimulación suelen ser de 1-3 V,

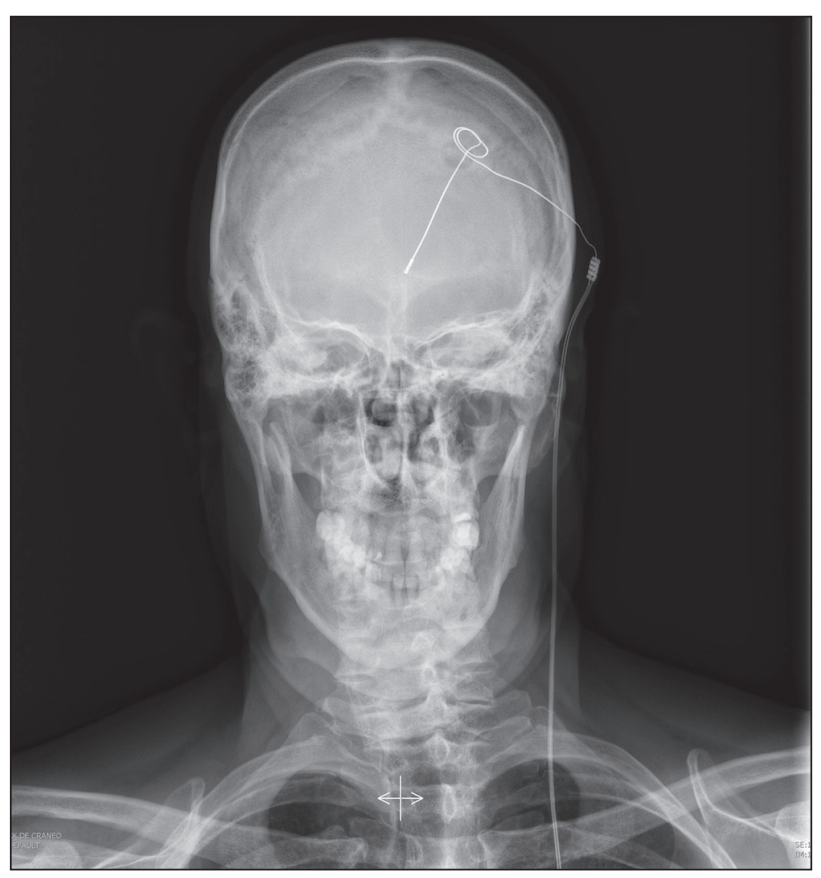

Fig. 3. Electrodo de estimulación cerebral profunda en zona del tálamo somato-sensorial.
50-100 Hz de frecuencia y 0,5 mseg de amplitud de pulso, siendo ligeramente más bajos en la estimulación de la sustancia gris periacueductal y periventricular.

En los casos con efecto positivo y tras un periodo de estimulación de prueba que suele oscilar entre los 7-15 días, se procede a la conexión con el generador y a la internalización del sistema. Los estimuladores utilizados son muy similares y emplean la misma tecnología que en la estimulación medular. La estimulación en general, se realiza en sesiones de 10-30 minutos varias veces al día.

El mecanismo de acción no se conoce completamente aunque se postulan como hipótesis, una estimulación de las vías inhibitorias corticofugales capaces de inhibir la transmisión del estímulo doloroso en el caso de la estimulación talámica y de la cápsula, y una liberación de opioides endógenos, junto con una activación del tálamo medial y la parte anterior del cíngulo en la estimulación de las otras estructuras (36). La estimulación hipotalámica es capaz de modular la actividad del núcleo descendente del trigémino influyendo así en el reflejo trigeminal causante de la cefalea en racimos (35).

Las indicaciones más claras de la técnica son el dolor talámico central, la lesión del plexo braquial, el miembro doloroso fantasma, la neuropatía periférica y el síndrome de cirugía de espalda fallida. En general se puede afirmar que los dolores nociceptivos responden mejor a la estimulación cerebral profunda que los neuropáticos (37).

Los diferentes estudios recogen un $80 \%$ de buenos resultados a largo plazo en síndromes dolorosos de cirugía de espalda fallida, con una reducción mayor del 50-60\% en la intensidad del dolor, una reducción de la ingesta de fármacos analgésicos, fundamentalmente opiáceos y una vuelta a su actividad física anterior en muchos de ellos. En ocasiones, y para obtener resultados positivos en este tipo de síndromes, es necesaria la combinación de dos o más electrodos intracerebrales en un mismo paciente, uno situado a nivel del tálamo sensorial, con el que se trataría fundamentalmente el dolor neuropático radicular de las extremidades, y otro a nivel de la sustancia gris periventricular o periacueductal con el que se trataría el dolor nociceptivo axial lumbar de estos pacientes $(37,38)$.

El índice de complicaciones de la estimulación cerebral profunda se sitúa en torno al $20 \%$ y se concretan en infecciones e intolerancia del material de estimulación, complicaciones neurológicas (hemorragias y alteraciones oculares), migración y fractura del electrodo, y aparición de disestesias en zonas que previamente estaban exentas de ellas (38).

\section{Estimulación cortical rolándica}

De reciente incorporación en el arsenal terapéutico antiálgico, la estimulación de la corteza motora introducida por Tsubokawa en 1991 (39), es en la actualidad una mo- 
dalidad de tratamiento especialmente indicada en el dolor crónico por deaferentización refractario al tratamiento conservador y que no ha podido ser tratado con otras modalidades de neuroestimulación o estas han sido inefectivas.

Este tipo específico de estimulación requiere la realización de una pequeña craneotomía y la localización por neuronavegación de la zona motora primaria contralateral a la zona dolorosa. El lugar ideal de estimulación se determina fácilmente desde las imágenes cerebrales del propio paciente y en función de la representación somatotópica de la región. En general se requiere la localización electroneurofisiológica de la zona motora elegida mediante potenciales evocados somatosensoriales y motores. Para la estimulación se utilizan dos electrodos planos de cuatro contactos que se disponen en paralelo y perpendiculares a la cisura de Rolando que se suturan a la duramadre (Fig. 4). Como en otras modalidades de neuroestimulación se realiza un periodo de prueba para valorar el grado de analgesia, como paso previo a la implantación definitiva del generador eléctrico del sistema. Los parámetros eléctricos disponibles en el manejo de estos equipos son idénticos a los utilizados en la estimulación medular.

Se especula con la posibilidad de reducción, mediante la estimulación del córtex motor, de la hiperactividad de las neuronas de la vía sensitiva recogida por encima de la lesión, como su mecanismo de acción (40).

Hasta el momento se han recogido resultados esperanzadores, esto es con efectos antiálgicos mayores del 50\% en más del 70\% de los pacientes, en el dolor que sigue a las lesiones medulares, a las avulsiones de plexo braquial, neuralgias faciales, neuropatías periféricas, miembro doloroso fantasma y dolor neuropático central derivado de un infarto isquémico o hemorrágico (40). En la actualidad no existen evidencias clínicas del empleo de la estimulación cortical en cuadros de origen raquídeo, salvo los relacionados con los lesionados medulares, ni en los síndromes de cirugía fallida de espalda, no obstante, los buenos resultados obtenidos en neuropatías periféricas y otros procesos neuropáticos, en buena lógica, pueden hacer pensar que se está ante una técnica que al igual que la estimulación cerebral profunda, podría utilizarse cuando cualquier tipo de aproximación terapéutica, incluidas las diferentes variantes de neuromodulación eléctrica o química, hubiese fracasado.

Las complicaciones registradas son poco frecuentes y quedan reducidas a la aparición de hematomas epidurales, infección del sistema y desarrollo de crisis epilépticas peroperatorias o durante el periodo de prueba.

\section{CONCLUSIÓN}

A lo largo de todo el presente artículo se ha pretendido dar relevancia a unos síndromes dolorosos, los originados

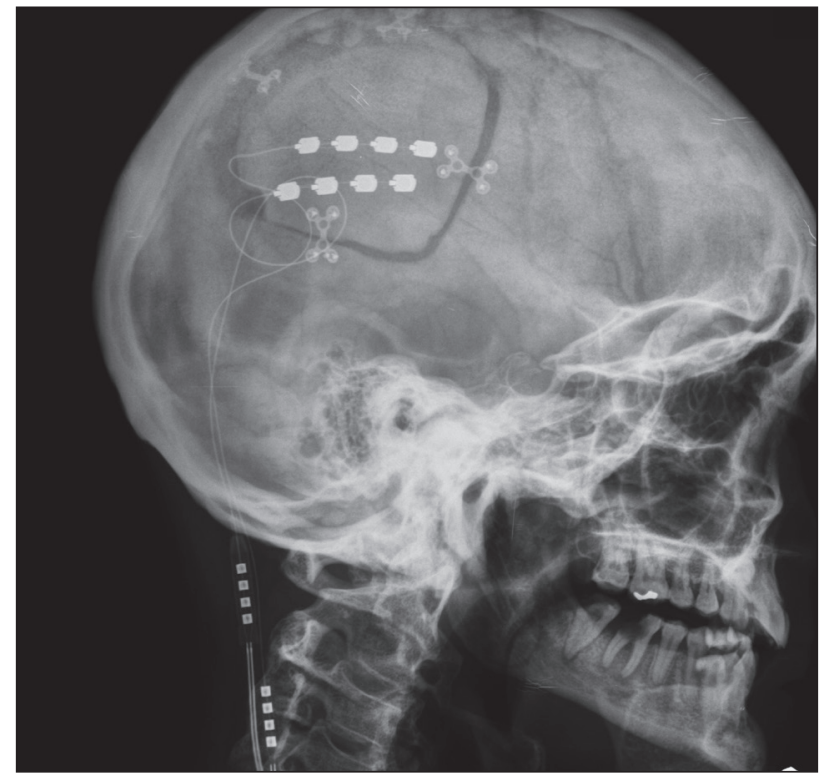

Fig. 4. Disposición de dos electrodos de cuatro contactos para estimulación cortical rolándica.

en el raquis, que cada día están teniendo una importancia capital en la medicina y sistemas sanitarios actuales, de ahí la preocupación, investigación y búsqueda permanente de un sistema terapéutico eficiente y eficaz que solucione estos problemas. El dolor raquídeo de siempre ha sido abordado desde otras perspectivas, más o menos invasivas, y más o menos esperanzadoras.

La estimulación eléctrica del sistema nervioso, de la que se ha intentado dar una visión general y objetiva en cuanto a indicaciones y resultados en este trabajo, tiene una vigencia en el tratamiento de los problemas raquídeos de más de cuarenta años. Ahora bien, la bondad y reversibilidad de las diferentes técnicas junto con los avances y desarrollo tecnológico tanto en generadores (totalmente implantables y recargables) como en electrodos (multicontacto que amplían el campo de parestesias eléctricas) han hecho que se facilite el procedimiento quirúrgico de implantación de los sistemas y se mejoren los resultados clínicos, haciendo que en la actualidad las diferentes modalidades de neuromodulación eléctrica hayan sido incorporadas definitivamente en el armamentarium terapéutico del dolor raquídeo y sobre todo del que sigue a la cirugía fallida de espalda.

Los diferentes estudios de revisión clínica y meta-análisis de estas técnicas sugieren que existe una evidencia de clase II en cuanto a eficacia y efectividad en el control de la sintomatología y en la mejora de la capacidad funcional y calidad de vida de los pacientes, teniendo un índice de recomendación de nivel B (será probablemente efectiva). 
La modulación eléctrica de diferentes estructuras del sistema nervioso, según los estudios de evidencia clínica de los múltiples autores que se han revisado aquí, se está mostrando como una terapia segura, eficaz y satisfactoria en el tratamiento de los cuadros de dolor incoercible originados en los diferentes niveles raquídeos.

\section{CORRESPONDENCIA:}

Guillermo García-March

Servicio de Neurocirugía

Hospital Clínico Universitario

Avda. Blasco Ibáñez, 17

46010 Valencia

e-mail: garciamarch@msn.com

\section{BIBLIOGRAFÍA}

1. Bonica JJ. Neck pain. In: Bonica JJ, editor. The Management of Pain. 2nd ed. Philadelphia: Lea \& Febiger; 1990.

2. Montero-Matamala A, Matute M. Dolor lumbar: exploración física y neurológica. Rev Soc Esp Dolor 2012;19(Supl. I):7-8.

3. Van Kleef M, Sluijter ME. Radiofrequency lesions in the treatment of pain of spinal origin. In: Gildenberg PL, Tasker RR, editors. Textbook of Stereotactic and Functional Neurosurgery. New York: McGraw-Hill; 1998.

4. Chan CW, Peng P. Failed back surgery syndrome. Review article. Pain Medicine 2011;12:577-606.

5. Hernández-Guinea BD, Hernández-Santos JR, Tenopala S, Canseco CP, Torres JC. Eficacia de la aplicación de ozono epidural y paravertebral a una concentración de $30 \mu \mathrm{g} / \mathrm{ml}$ para el manejo de dolor crónico en pacientes con síndrome de cirugía fallida de espalda. Rev Soc Esp Dolor 2012;19 (1):3-10.

6. Kumar K, Taylor RS, Jacques L, et al. Spinal cord stimulation versus conventional medical management for neuropathic pain: A multicentre randomised controlled trial in patients with failed back surgery syndrome. Pain 2007;132:179-88.

7. Van Buyten JP, Linderoth B. The failed back surgery syndrome: Definition and therapeutic algorithms - An update. Eur J Pain 2010;(Supl. 4):273-86.

8. Busquets J. Síndrome de cirugía fallida de espalda. Rev Soc Esp Dolor 2012;19(Supl. I):15.

9. Kellaway P. The part placed by electrical fish in the early history of bioelectricity and electrotherapy. Bull Hist Med 1946;20:112-37.

10. Stillings D. A survey of the history of electrical stimulation for pain to 1900. Med Instrum 1975;9:235-59.

11. Melzack R, Wall PD. Pain mechanisms: A new theory. Science 1965;150:971-9.

12. Pert CD, Snyder SH. Opiate receptor. Demostration in nervous tissue. Science 1973;179:1011-14.

13. Terenius L. Sterospecific interaction between narcotic analgesics and synaptic plasma membrane fraction of rat cerebral cortex. Acta Pharmacol Toxicol 1973;32:317-20.
14. Wall PD, Sweet WH. Temporary abolition of pain in man. Science 1967;155:108-9.

15. Shealy CN, Mortimer JT, Reswick J. Electrical inhibition of pain by stimulation of the dorsal column: Preliminary clinical reports. Anesth Analg 1967;46:489-91.

16. Mazars G, Merienne L, Ciocola C. Stimulations thalamiques intermitentes antalgiques: Note preliminaire. Rev Neurologiq 1973;128:273-9.

17. Richardson DE, Akil H. Pain reduction by electrical stimulation in man. J Neurosurg 1977;47:178-94.

18. Gol A. Relief of pain by electrical stimulation of the septal area. J Neurol Sci 1967;5:115-20.

19. Fields HL, Basbaum AI. Endogenous pain control mechanisms. In: Wall PD, Melzack R, editors. Textbook of Pain. Edimburgh: Churchill Livingstone; 1984.

20. Sánchez-Ledesma MJ, García-March G, Blasco C, Broseta J. Uso terapéutico de la estimulación eléctrica transcutánea. En: Barbera J, editor. Estimulación eléctrica biológica. Cádiz: Servicio de Publicaciones de la Universidad de Cádiz; 1989.

21. Carroll D, Moore RA, MsQuay HJ, Fairman F, Tramer M, Leijon G. Transcutaneous electrical nerve stimulation (TENS) for chronic pain. Cochrane Database Syst Rev 2001;3:CD003222.

22. Ortega E. El valor de la estimulación eléctrica transcutánea. Rev Soc Esp Dolor 2010;17(Espec Cong 1):48-9.

23. Sluka KA, Walsh D. Transcutaneous electrical nerve stimulation: Basic science mechanisms and clinical effectiveness. J Pain 2003;4:109-21.

24. Abejón D, Del Saz J, Calvo R. Estimulación subcutánea. Rev Soc Esp Dolor 2010;17(Espec Cong 1):50-1.

25. Mironer YE, Hutcheson JK, Satterthwaite JR, LaTourette PC. Prospective, two-part study of the interaction between spinal cord stimulation and peripheral nerve field stimulation in patients with low back pain: Development of a new spinal-peripheral neuroestimulation method. Neuromod 2011;14(2):151-4.

26. Hosobuchi Y, Adams JE, Weinstein PR. Preliminary percutaneous dorsal columna stimulation prior to permanente implantation. Technical note. J Neurosurg 1972;37:242-5.

27. García-Pérez ML, Badenes R, García-March G, Bordes V, Belda FJ. Epidural anesthesia for laminectomy lead placement in spinal cord stimulation. Anesth Analg 2007;105:1458-61.

28. Barolat G, Massaro F, He J, Zeme S, Ketcik B. Mapping of sensory responses to epidural stimulation of intraspinal neural structures in man. J Neurosurg 1993;78:233-9.

29. Van Buyten JP. Tratamiento con sistemas de EEM de alta frecuencia en el dolor lumbar. In IX Congreso de la Sociedad Española del Dolor. Barcelona, España, 6-9 de Junio de 2012.

30. Meyerson BA, Linderoth B. Mode of action of spinal cord stimulation in neuropathic pain. J Pain Symptom Manage 2006;31:S6-S12.

31. North RB, Kidd DH, Farrokhi F, Piantadosi SA. Spinal cord stimulation versus repeated lumbosacral spine surgery for chronic pain: A randomized, controlled trial. Neurosurgery 2005;56:98-107.

32. Taylor RS, Van Buyten JP, Buchser E. Spinal cord stimulation for chronic back and leg pain and failed back surgery syndrome: a systematic review and analysis of prognostic factors. Spine 2005;30:152-60. 
33. Pallarés JJ. Tratamiento del SCFE lumbar por neuroestimulación. Rev Soc Esp Dolor 2012;19(Supl. I):19-21.

34. García-March, G. Tratamiento con EEM y electrodos planos multihilera en el dolor lumbar. In IX Congreso de la Sociedad Española del Dolor. Barcelona, España, 6-9 de junio de 2012.

35. Leone M. Deep brain stimulation in headache. Lancet Neurol 2006;5:873-7.

36. Hamani C, Fontaine D, Lozano A. DBS for persistent noncancer pain. In: Lozano A, Gildenberg PL, Tasker RR, editors. Textbook of stereotactic and functional neurosurgery. $2^{\mathrm{a}}$ ed. Berlin: Springer; 2009.
37. Bittar RG, Kar-Purkayastha I, Owen SL, Bear R, Green A, Wang S, et al. Deep brain stimulation for pain relief: A metaanalysis. J Clin Neurosci 2005;12:515-9.

38. Young RF. Deep brain stimulation for failed back syndrome. In: Gildenberg PL, Tasker RR, editors. Textbook of Stereotactic and Functional Neurosurgery. New York: McGraw-Hill; 1998.

39. Tsubokawa T, Katayama Y, Yamamoto Y, Hirayama T, Koyama S. Chronic motor cortex stimulation for the treatment of central pain. Acta Neurochir Suppl 1991;52:137-9.

40. Nguyen JP, Lefaucheur JP, Raoul S, Roualdes V, Péréon Y, Keravel Y. Motor cortex stimulation for the treatment of neurophatic pain. In: Krames ES, Peckham PH, Rezai AR, editors. Neuromodulation. Amsterdam: Elsevier; 2009. 